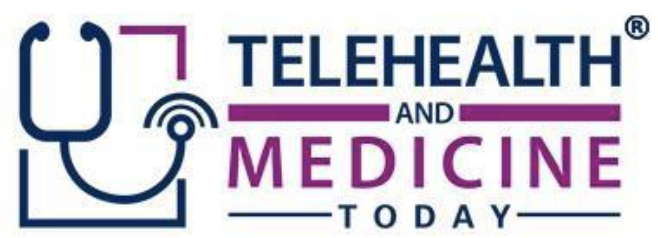

\title{
Telemental and Telebehavioral Health Considerations: A 50-State Analysis on the Development of Telehealth Policy
}

\author{
Amy F. Lerman, ${ }^{1}$ Daniel Kim, ${ }^{1}$ Francesca R. Ozinal, ${ }^{1}$ Theresa E. Thompson ${ }^{1}$
}

Author Affiliation: ${ }^{1}$ Epstein, Becker, Green, P.C.

Corresponding Author Amy F. Lerman, ALerman@ebglaw.com.

Keywords: Telehealth, Telemental, Telebehavioral, Behavioral, Technology

Section: Review

Contemporary healthcare delivery models have

a patient-centered approach, a focus on integrating the various types of services patients need, an emphasis on interdisciplinary treatment teams, and a commitment to adopting innovative technologies. Increasing utilization of telehealth technologies has helped foster these contemporary service delivery models by improving access to healthcare services, leveraging the expertise of specialty providers to the point-of-service, and enhancing opportunities for healthcare professional and consumer education. As such, telehealth delivery models have become mainstream and viable solution for healthcare providers, physicians, and employers. According to an August 2016 report from the U.S. Department of Health and Human Services, an estimated $61 \%$ of healthcare institutions use some form of telehealth technology, and between $40 \%$ and $50 \%$ of all hospitals in the United States employ some form of telehealth technology. ${ }^{1}$
Following suit, the provision of telebehavioral or "telemental" health services continue to trend upward, as behavioral health providers become more interested in utilizing telehealth platforms to connect with their patients. Mental illness affects millions of individuals in the U.S., from all walks of life and across all age groups, contributing significantly to the burdens of disease. ${ }^{2}$ While mental illness can be recurrent and serious, often it is treatable, provided affected individuals have access to necessary resources. Patients surveyed regarding their use of telemental health services express consistent and affirmative beliefs that telemental health services provide positive benefits. Surveys report little or no difference in levels of patient satisfaction compared to face-to-face interactions between patients and their behavioral health providers. ${ }^{3,4}$

As states continue to support and expand the exciting benefits telehealth technologies and services offer, our legislators, healthcare systems, and regulatory bodies are being tasked 
with working together to navigate the numerous legal and regulatory issues that come with it. Epstein Becker Green ("EBG") recently released an Appendix to its "50-State Survey of Telemental/Telebehavioral Health (2016), " with new and updated analysis on the laws, regulations, and regulatory policies affecting the practice of telemental/telebehavioral health in all 50 states and the District of Columbia. ${ }^{5}$ The 2017 Appendix reflects the incredibly active legislative efforts in most states with respect to the provision of telehealth services. In fact, only two states, Connecticut and Massachusetts, have not made changes to their legal and regulatory framework since the 2016 Survey. This suggests a lot of policy activity among states to regulate and provide meaningful guidance to telehealth providers.

\section{NTRODUCTION TO THE PROBLEM Shortage of Behavioral Health Services in the U.S.}

Despite significant prevalence of mental illness, the U.S. healthcare system largely has failed to address the shortage of behavioral health services needed to respond to the burdens of mental illness. ${ }^{5}$ Historically, the gap in untreated serious mental illness costs the U.S. $\$ 300$ billion annually in total lost costs. ${ }^{5}$ Furthermore, there is a shortage of healthcare providers who are appropriately qualified to provide needed services, Presently, there is only one qualified behavioral health provider for every 790 individuals and approximately 5,000 mental health, Health Professional Shortage Areas ("HPSAs"). 6,7 This means that approximately 3,000 additional psychiatrists are needed to eliminate current HPSA designations. ${ }^{8}$ Moreover, behavioral health providers are unevenly distributed across the U.S., with the majority of these providers concentrated in the Northeast.

\section{Telehealth's Contribution in Filling Gap} Coverage

Constant development of new technologies significantly contributes to the recent boom in the telehealth industry, due in large part to the development of mobile health applications that promote easy and secure access to healthcare providers. ${ }^{5}$ Although both federal and state lawmakers' legislative efforts have helped increase access to healthcare providers via telehealth technology for populations living in rural areas, recent natural disasters, such as hurricanes Harvey and Irma, show that the benefits of telehealth go beyond just meeting the healthcare needs of the population in rural areas. $^{9}$

\section{Efficacy of Telemental Health}

Integration of telehealth technologies into the provision of behavioral health services makes sense because the use of these technologies (e.g., real-time video, audio conferencing) does not meaningfully change the basic premise of traditional behavioral healthcare delivery models. Rarely, for example, do behavioral health providers interact physically with their patients during in-person encounters, so transitioning these encounters to occurring in a virtual setting does not significantly change the way these services are delivered. Patients seeking treatment from telemental health providers may do so in the comfort of their own home, thereby minimizing the stigma that may be associated with physically visiting a behavioral health provider in-person. By minimizing the stigmatization that sometimes is associated with a person seeking mental health treatment, a telehealth platform may encourage patients to seek out behavioral counseling from mental health providers more frequently and consistently, enabling mental health providers to better diagnose and provide treatment accordingly to these patients. As a result, this could improve the quality of mental health 
services rendered, in addition to improving access to behavioral health providers in parts of the country where there are shortages or even a complete absence of qualified behavioral health providers.

\section{Challenges to Greater Adoption and Utilization of Telemental Health Services}

Despite the positive potential and benefits associated with provision of telemental health services, actual adoption and utilization of these services is still relatively low. ${ }^{10}$ This is due, in part, to the complex and often inconsistent regulatory environment in which all telehealth providers operate. At the federal level, there has been limited legislative success in expanding access to (i.e., coverage and reimbursement for) telehealth services. Historically and presently, federal healthcare programs, including Medicare, only cover certain telehealth services and do so under fairly limited conditions (e.g., geographic restrictions, service types, etc.). Federal efforts to improve coverage and reimbursement opportunities for telehealth providers only recently have gained any meaningful momentum. On November 7, 2017, the House of Representatives passed the Veterans E-health \& Telemedicine Support ("VETS") Act of 2017 that would allow the Department of Veterans Affairs ("VA") to deliver care via telehealth to its patients without geographic restrictions. ${ }^{11}$ The Veterans Community Care and Access Act of 2017, introduced on December 4, 2017, contains similar language to the VETS Act. ${ }^{12}$ These efforts for the federal government to expand their adoption of telehealth have received support from a broad range of organizations, including the American Telemedicine Association, Federal Trade Commission, American Academy of Family Physicians, American Psychological Association, and many provider groups. However, despite many similar efforts, and unlike comparable state level counterparts, telehealth legislation at the Federal level often loses momentum after it is introduced. ${ }^{13}$

\section{REMOTE PRESCRIBING CHALLENGES}

Another significant barrier to greater adoption and utilization of telehealth services is the Ryan Haight Online Pharmacy Consumer Protection Act of 2008 ("Ryan Haight Act"). The law was enacted following the unfortunate and untimely death of Ryan Haight, an 18-year old student who overdosed on prescription pain killers delivered to his door by an internet pharmacy based on a prescription written by a physician he had never seen. The Ryan Haight Act amended the federal Controlled Substances Act by requiring prescribing practitioners to be physically present when prescribing or allowing the prescription of controlled substances by remote practitioners only if the prescribing practitioner previously conducted an in-person physical assessment of the patient. Although states have started to examine and refine their own remote prescribing laws - even in some cases permitting (under appropriate circumstances) the remote prescribing of controlled substances - the Ryan Haight Act remains in place and is considered a significant obstacle to the regulatory progress of telehealth.

Finally, there is a lack of cohesion among state telehealth laws and regulations, as states continue actively legislating to regulate the practice of telehealth within their borders. For example, Hawaii allows telehealth providers to prescribe controlled substances only after a provider has performed an in-person consultation. However, Delaware allows telehealth providers to prescribe controlled substances without the in-person requirement. Without any comparable federal oversight of telehealth providers and services, an understanding of the regulatory obligations of providers requires a state-by-state analysis. The 
state-to-state variations in these laws, from coverage and reimbursement issues to practitioner-patient relationship definitions, contribute to the slower than expected expansion of telehealth among healthcare systems and provider groups.

\section{METHODOLOGY}

Epstein Becker Green's 2016 Survey and 2017

Appendix are extensive compilations of state research regarding the laws, regulations, and regulatory policies impacting provision of telemental health services in all 50 states and the District of Columbia. The original concept for the 2016 Survey was to examine the rapid growth of telebehavioral health services by looking at the increasingly complex legal issues associated with this trend, and the 2017 Appendix has supplemented EBG's original analysis.

The research consists of a review of state statutes and regulations, as well as state Medicaid program materials (e.g., provider billing and benefit manuals and program coverage policies). It also examines a wide variety of state-focused guidance published by various professional boards, including Boards of Medicine, Psychology, Social Work, Professional Counselors, Therapists, Nurses, and Pharmacy (related to remote prescribing). In certain states, guidance published by Departments of Health and/or Education (as related to professional licensure) also is reviewed.

While provision of telebehavioral health services touches on some federal laws and regulations (e.g., the Health Insurance Portability and Accountability Act), most of the significant legal and regulatory issues involve state laws. But, because states have taken varying approaches to regulating telehealth/telemedicine services generally and telebehavioral health services specifically, this has resulted in an inconsistent patchwork of laws that can vary greatly from state to state.

The 2016 Survey and 2017 Appendix provide summaries of relevant state laws and guidance related to such issues as how terms like "telehealth," "telemedicine," and "telebehavioral health" are defined, how provider-patient relationships may be established via telehealth, acceptable modalities for provision of telehealth services, and providers' prescribing authority. Variance between the guidance provided by states is extremely common and dependent on the types of resources available. While some guidance provided by states is extremely clear, available guidance in other states leaves significant room for interpretation.

\section{RESULTS}

Since the original research was released, there have been significant changes in the ways states are approaching telehealth legislation and regulation. For example, there is no longer a single state that requires physicians meet with a patient in-person before they provide telemedicine services. However, at least one state, Arkansas, requires that other types of providers (e.g., psychologists, counselors, advanced practice registered nurses [APRNs]) conduct an in-person exam before rendering telehealth services to a patient. Professional boards in some other states (e.g., Colorado and Massachusetts) recommend an initial face-toface evaluation by other types of providers (e.g., psychologists, social workers), more as a best practice. Another significant change is the nature of the legislation being enacted. An abundance of the activity over the last two years has involved states incorporating telemedicine and telehealth deeply into their Medicaid programs, expanding coverage and reimbursement for various services and providers. Fifteen states made changes to their Medicaid policies to 
address various telehealth issues. Further, as the field of telehealth continues to develop, states are adapting their understanding of it. Since the initial publication of the 2016 Survey, nineteen states have defined telemedicine, telehealth, or a subcategory, or updated their telemedicine or telehealth definitions to broader and encompassing language. As discussed in greater detail below, however, while the language used to regulate telehealth broadens, the detail of the regulations becomes more specific, addressing more specific care situations, providers, and payors.

\section{Regional Commonalities}

The change happening through state regulation is sporadic, with some states developing quickly and some only recently catching up. A review of the results of the 2016 Survey did not yield any particularly meaningful patterns in terms of regional commonalities among states. For example, EBG did not observe that a particular region of the country, such as the Southeast or the Pacific Northwest, had updated its telemental health laws, regulations, or regulatory policies in a consistent manner.

However, most states made some change to the laws or regulations governing telemental health providers. A few states seemed to completely overhaul their telehealth policy since the publication of the initial survey, including Georgia, Hawaii, Idaho, New Jersey, New York, and Texas. Conversely, a handful of states made no significant changes, or any changes at all, to their laws, regulations, or regulatory policies affecting the provision of telemental health services. Connecticut and Massachusetts made no substantive changes to their telehealth laws since the original survey. Additionally, California, Illinois, and Pennsylvania made limited changes.

\section{Patterns Within Subcategories of Providers}

While there were not many regional commonalities among the states, EBG observed several meaningful patterns with respect to how states have updated the statutory and regulatory schemes governing telemental health providers between the publication of the 2016 Survey and 2017 Appendix. Many of these patterns relate to the criteria states have put in place for establishing practitioner-patient relationships via telemedicine or telehealth.

Prior to rendering telehealth or telemedicine services, many states require providers to:

- Confirm the patient's identity and location;

- Disclose to the patient the provider's identity and credentials;

- Provide the patient with information concerning follow-up care, when appropriate;

- Inform the patient regarding limitations, risks, and benefits associated with the services; and

- Obtain from the patient all necessary informed consent.

With respect to psychologists, eight states have new or revised definitions or standards for the establishment of the provider-patient relationship. But only one of these, Arkansas, requires psychologists to conduct an in-person examination prior to the provision of telemedicine services in order to establish an appropriate professional relationship with the patient. Obtaining a patient's informed consent prior to providing telebehavioral health services also is a common criterion for establishing a psychologist-patient relationship. Additionally, several states have delineated the acceptable modalities for the practice of telepsychology since the original survey, including Arkansas, Idaho, Maine, New Jersey, and Rhode Island. 
With respect to psychiatrists, the most common changes EBG observed were relative to the prescribing requirements. Twelve states made changes to their controlled substances policies, often altering prescription rules or specifically identifying certain substances, such as opioids, that were explicitly prohibited from being prescribed via telemedicine. Some states, such as Michigan, permitted prescribers to prescribe controlled substances via telemedicine, although this is still prohibited by federal law, as discussed above. Other states used the last two years to clarify their rules. For example, within this past year, Oklahoma specified "certain requirements" to mean "equipment requirements" that prescribers had to satisfy if they wish to prescribe via telemedicine.

For counselors, the most noticeable change states made to the manner in which counselors provide telemedicine services relates to the restrictions on counselors' scope of practice. Since the original survey, eleven states either revised these restrictions or adopted new scope of practice restrictions on counselors providing telemedicine services. For example, at the time of publication of the original survey, Idaho placed the same restrictions on counselors providing telemedicine services as those it placed on psychiatrists. More recently, the Idaho Board of Professional Counselors and Marriage and Family Therapists weighed in on the issue and provided scope of practice restrictions specific to counselors. These restrictions are separate and apart from those dealing with psychiatrists. Moreover, New Jersey had not provided any clear guidance for counselors at the time of the original survey, but recently placed certain restrictions on the scope of practice for healthcare providers, including professional counselors who practice via telemedicine.
Several patterns also emerged amongst advance practice registered nurses ("APRNs"). First, and similar to psychologists, five states revised practitioner-patient relationship requirements. Only one state, Arkansas, requires APRNs to conduct an in-person physical exam prior to the provision of telemedicine services to establish the practitioner-patient relationship. Second, seven states have placed new or revised scope of practice restrictions on APRNs providing telemedicine services. Third, eight states have new licensing requirements specific to telemedicine for APRNs.

\section{Meaning of Findings}

The 2016 Survey and 2017 Appendix demonstrate that there is an abundance of new and updated guidance from states across the country that affects, and even changes, the practice of telemental health services. Among the key findings is that while telehealth-focused regulations applicable to physicians (e.g., psychiatrists) are still the most prevalent, states have started to expand their regulatory frameworks with respect to other types of behavioral health providers, including psychologists, social workers, and counselors. In New Jersey, for example, the enactment of a new telemedicine law includes a fairly broad definition of the term "healthcare provider," indicative of a movement toward greater acceptance of different provider types (i.e., not just physicians) utilizing telehealth technologies to care for their patients.

Healthcare practitioners continue to examine the business case for incorporating telehealth into their care delivery models. An advantage of incorporating telehealth technologies into health practice is the continuity of care that virtual access to patients affords providers, as well as the greater sense of connection that patients feel 
as a result. Particularly in situations where patient's healthcare costs are prepaid, or when providers are paid for care coordination activities, use of telehealth technologies can provide significant benefits because traditional patient visits can be time-intensive, and many of the typical needs of patients (e.g., answering questions, delivering basic primary care services) can be addressed efficiently through telehealth.

However, the regulatory environment in which healthcare providers operate must be conducive to establishing these virtual care delivery models. Fortunately, many states are working diligently to remove some of the traditional regulatory and policy barriers related to where telehealth services may occur and how providerpatient relationships may be established (often a prerequisite to actually performing the telehealth services). States are delving with more specificity into regulatory parameters related to the provision of telebehavioral healthcare services, giving behavioral health providers a clearer understanding of the rules and requirements specific to "telepsychiatry" (or the like) and not just telehealth/telemedicine more generally. The significant policy developments that occurred this past year indicate telehealth is becoming a staple of behavioral healthcare, and the healthcare system should anticipate further changes moving forward.

\section{Acknowledgement: N/A}

Funding Statement There was no public or private funding provided in the creation of this work.

\section{Conflict of Interest: None}

\section{Contributors}

To fulfil all of the criteria for authorship, every author of the manuscript has made substantial contributions to ALL of the work and participated sufficiently in the work to take public responsibility.

Copyright ownership: Amy F. Lerman

\section{REFERENCES}

1. U.S. Dept. of Health and Human Services. Report to Congress e-health and telemedicine [Internet]. 2016 Aug [cited 2018 Jan 18]; 4. URL:

https://aspe.hhs.gov/system/files/pdf/20 6751/TelemedicineE-HealthReport.pdf.

2. Elizabeth RW. Mortality in mental disorders and global disease burden implications. JAMA Psychiatry. 2014;72:335-342.

3. Donald MH, Daphne CF, Michelle BP, et al. The effectiveness of telemental health: a 2013 review. Telemed J E Heath. 2013 Jun;19:444-54.

4. Amy N, A new emphasis on telehealth. Monitor on Psychology. 2011 June; 42(6):40. URL: http://www.apa.org/monitor/2011/06/tel ehealth.aspx

5. Epstein Becker \& Green, P.C. 50-state survey of telemental/telebehavior health. 2016 June;1:6.n URL: https://www.ebglaw.com/content/upload s/2017/10/EPSTEIN-BECKERGREEN-2017-APPENDIX-50-STATETELEMENTAL-HEALTHSURVEY.pdf

6. Leslie G. Even with coverage expansion, access to mental health services poses challenges. Kaiser Health News [Internet]. 2014 Dec [cited 2018 Jan 18]. URL: https://khn.org/news/even-withcoverage-expansion-access-to-mentalhealth-services-poses-challenges/.

7. Health Resources \& Services Administration Data Warehouse. Shortage areas [Internet].[cited 2018 Jan 17] URL: 
https://datawarehouse.hrsa.gov/topics/sh ortageAreas.aspx

8. Bruce J. U.S. psychiatrist shortage intensifies [Internet]. Forbes. 2017 Jan 6 [cited 2018 Jan 18]. URL https://www.forbes.com/sites/brucejapse n/2017/06/06/psychiatrist-shortageintensifies/\#9d88eca5d964.

9. Eric W. Harvey's aftermath brings mHealth, telehealth to the forefront.

mHealthIntelligence [Internet]. 2017

Aug [cited 2018 Jan 18]. Available from:

https://mhealthintelligence.com/news/ha rveys-aftermath-brings-mhealthtelehealth-to-the-forefront.

10. Rachel ZA, Limited reimbursement hinders telehealth adoption. Modern Healthcare [Internet]. 2017 Oct [cited 2018 Jan 18]. URL:

http://www.modernhealthcare.com/articl e/20171010/NEWS/171019998.

11. Rachel ZA. House Votes to Ease VA Telemedicine Restrictions. Modern Healthcare [Internet]. 2017 Nov [cited 2018 Jan 18]. URL: http://www.modernhealthcare.com/articl e/20171107/NEWS/171109899.
12. Eric W. Senators include telemedicine in new bill to modernize the VA. mHealthIntelligence [Internet]. 2017 Dec [cited 2018 Jan 18]. URL: https://mhealthintelligence.com/news/se nators-include-telemedicine-in-new-billto-modernize-the-va.

13. Eric W. Congress is coming at CMS from all angles with telehealth bills. mHealthIntelligence [Internet]. $2017 \mathrm{Jul}$ [cited 2018 Jan 18]. URL: https://mhealthintelligence.com/news/co ngress-is-coming-at-cms-from-allangles-with-telehealth-bills.

\section{Copyright Ownership}

This is an open access article distributed in accordance with the Creative Commons Attribution Non-Commercial (CC BY-NC 4.0) license, which permits others to distribute, adapt, enhance this work noncommercially, and license their derivative works on different terms, provided the original work is properly cited as first published in Telehealth and Medicine Today ${ }^{\mathrm{TM}}$, and the use is non-commercial. 